\title{
Topological degree theories for continuous perturbations of resolvent compact maximal monotone operators, existence theorems and applications
}

\author{
Teffera M. Asfaw
}

Department of Mathematics, Virginia Polytechnic Institute and State University, Blacksburg, VA 24061, USA.

\begin{abstract}
Let $X$ be a real locally uniformly convex reflexive Banach space. Let $T: X \supseteq D(T) \rightarrow 2^{X^{*}}$ and $A: X \supseteq D(A) \rightarrow 2^{X^{*}}$ be maximal monotone operators such that $T$ is of compact resolvents and $A$ is strongly quasibounded, and $C: X \supseteq D(C) \rightarrow X^{*}$ be a bounded and continuous operator with $\mathrm{D}(\mathrm{A}) \subseteq \mathrm{D}(\mathrm{C})$ or $\mathrm{D}(\mathrm{C})=\overline{\mathrm{U}}$. The set $\mathrm{U}$ is a nonempty and open (possibly unbounded) subset of $X$. New degree mappings are constructed for operators of the type $T+A+C$. The operator $C$ is neither pseudomonotone type nor defined everywhere. The theory for the case $\mathrm{D}(\mathrm{C})=\overline{\mathrm{U}}$ presents a new degree mapping for possibly unbounded $\mathrm{U}$ and both of these theories are new even when $\mathrm{A}$ is identically zero. New existence theorems are derived. The existence theorems are applied to prove the existence of a solution for a nonlinear variational inequality problem.
\end{abstract}

Keywords: Compact resolvents, continuous operator, degree theory, variational inequality, homotopy invariance, maximal monotone.

2010 MSC: 47H11, 47H14, 47H07.

(C)2020 All rights reserved.

\section{Introduction and preliminaries}

Topological degree theories have been essential tools in the study of existence of solutions for equation and inequality problems arising from different disciplines. Brouwer [12] degree theory for continuous functions on $\mathbb{R}^{N}$ and, Leray and Schauder [25] degree theory for compact displacement of the identity in real Banach spaces have been played important roles to derive corresponding surjectivity and fixed point results. The basic details of these degree theories can be found in the books by Lloyd [26] and, O'regan et al. [29], and in the survey paper by Mawhin [27], and references therein. In series of papers, Browder [14-16] developed degree theories for perturbed operators of monotone type in reflexive Banach spaces. The creation of this theory helped to deal with equations and inequalities involving operators defined from a reflexive Banach space into its dual space. A number of generalizations and/or improvements of Browder's theory have been developed over the past 45 years. The main contributions are due to $\mathrm{Hu}$

\footnotetext{
${ }^{*}$ Corresponding author

Email address: teffera6@vt.edu (Teffera M. Asfaw)
}

doi: $10.22436 /$ jnsa.013.05.02

Received: 2019-09-06 Revised: 2020-01-10 Accepted: 2020-01-16 
and Papageorgiou [18], Berkovits and Mustonnen [10], Kobayashi and Otani [22], Kartsatos and Skrypnik [19], Adhikari and Kartsatos [1], Asfaw and Kartsatos [7] and, Asfaw [3-5]. It is worth mentioning that Browder's theory and those in $[3,5,7,10,14,18,19,22]$ are for $\left(S_{+}\right)$(or variant of $\left(S_{+}\right)$) perturbations of maximal monotone operators. On the other hand, it is known due to Propositions 27.6 and 27.7 in the book by Zeidler [34] that the classes of continuous and pseudomonotone operators (e. g., $\left(S_{+}\right)$operators) are distinct and coincide only in finite dimensional spaces. The reader can find various examples of operators with compact resolvents in the books by Varabie [33], Showalter [32] and the references therein. The existence of problems involving continuous perturbations of resolvent compact operators and the nonexistence of a unified theory concerning this class of problems appeal a new theory. The present paper is devoted to develop new degree theories and derive applicable existence theorems.

In what follows, $X$ and $X^{*}$ denote a real reflexive Banach space and its dual space, respectively. The norms of these spaces will be denoted by $\|\cdot\|$. The symbol $\left\langle x^{*}, x\right\rangle$ denotes the value $x^{*}(x)$, where $x^{*} \in X^{*}$ and $x \in X$. For an operator $B: X \rightarrow 2^{X^{*}}$, the domain of $B$, denoted by $D(B)$, is

$$
\mathrm{D}(\mathrm{B})=\{x \in \mathrm{X}: \mathrm{B} x \neq \emptyset\},
$$

the range of $B$, denoted by $R(B)$, is understood as $R(B)=\cup_{x \in D(B)} B x$ and the graph of $B$, denoted by $\mathrm{G}(\mathrm{B})$, is

$$
\mathrm{G}(\mathrm{B})=\left\{\left(x, x^{*}\right): x \in \mathrm{D}(\mathrm{B}), x^{*} \in \mathrm{B} x\right\} .
$$

The operator B is "bounded" if it maps a bounded subset of $D(B)$ into a bounded subset of $X^{*}$. In addition, $B$ is called "quasibounded" if for each $M>0$, there exists $K(M)>0$ such that

$$
\left\langle w^{*}, x\right\rangle \leqslant M\|x\|, \quad \forall x \in \bar{B}_{M}(0), \text { and } w^{*} \in \mathrm{B} x,
$$

implies $\left\|w^{*}\right\| \leqslant K(M)$. The operator B is called "strongly quasibounded" if for each $M>0$ there exists $K(M)>0$ such that

$$
\left\langle w^{*}, x\right\rangle \leqslant M, \quad \forall x \in \overline{\mathrm{B}}_{M}(0) \text { and } w^{*} \in \mathrm{B} x,
$$

implies $\left\|w^{*}\right\| \leqslant K(M)$. A single-valued operator $\mathrm{F}: \mathrm{X} \supseteq \mathrm{D}(\mathrm{F}) \rightarrow \mathrm{X}^{*}$ is "compact" if it is continuous and maps each bounded subset $E$ of $D(F)$ into relatively compact subset of $X^{*}$ (i.e., $\overline{F(E)}$ is a compact subset of $\left.X^{*}\right)$.

Definition 1.1 is needed in the squeal.

Definition 1.1. An operator $T: X \supset D(T) \rightarrow 2^{X^{*}}$ is said to be

(a) "monotone" if

$$
\left\langle u^{*}-v^{*}, x-y\right\rangle \geqslant 0
$$

for all $\left(x, u^{*}\right)$ and $\left(y, v^{*}\right)$ in $G(T)$;

(b) "maximal monotone" if $\mathrm{T}$ is monotone and

$$
\left\langle u^{*}-u_{0}^{*}, x-x_{0}\right\rangle \geqslant 0,
$$

for every $\left(x, u^{*}\right) \in G(T)$ implies $x_{0} \in D(T)$ and $u_{0}^{*} \in T x_{0}$, i.e., $\left(x_{0}, u_{0}^{*}\right) \in G(T)$. Equivalently, $T$ is "maximal monotone" if and only if $T$ is monotone and $T+\lambda J$ is surjective for all $\lambda>0$ (c.f., Barbu [9, Theorem 2.2]);

(c) "resolvent compact maximal monotone" if $\mathrm{T}$ is maximal monotone and

$$
(\mathrm{T}+\lambda \mathrm{J})^{-1}: \mathrm{X}^{*} \rightarrow \mathrm{D}(\mathrm{T}),
$$

is a compact operator for each $\lambda>0$, equivalently for some $\lambda>0$; 
(d) "coercive" if either $\mathrm{D}(\mathrm{T})$ is bounded or there exists a function $\phi:[0, \infty) \rightarrow(-\infty, \infty)$ such that $\phi(t) \rightarrow \infty$ as $t \rightarrow \infty$ and

$$
\left\langle y^{*}, x\right\rangle \geqslant \phi(\|x\|)\|x\|,
$$

for all $x \in D(T)$ and $y^{*} \in T x$.

The mapping $\mathrm{J}: \mathrm{X} \rightarrow 2^{\mathrm{X}^{*}}$ given by

$$
J(x)=\left\{x^{*} \in X^{*}:\left\langle x^{*}, x\right\rangle=\|x\|^{2},\left\|x^{*}\right\|=\|x\|\right\},
$$

is known as the "normalized duality mapping". It is known due to Hahn-Banach theorem that $J(x) \neq \emptyset$ for all $x \in X$. The local uniform convexity of $X$ and $X^{*}$ implies that $J$ is single valued, bounded, monotone, bicontinuous and of type $\left(S_{+}\right)$, i.e., it is maximal monotone. A maximal monotone operator $\mathrm{T}$ has the property that

$$
(\mathrm{T}+\lambda \mathrm{J})^{-1}: \mathrm{X}^{*} \rightarrow \mathrm{D}(\mathrm{T}),
$$

is a single-valued, monotone and demicontinuous operator.

For each $\lambda>0$, the operators $T_{\lambda}: X \rightarrow X^{*}$, given by

$$
\mathrm{T}_{\lambda} x=\left(\mathrm{T}^{-1}+\lambda \mathrm{J}^{-1}\right)^{-1} x,
$$

and $\mathrm{J}_{\lambda}: \mathrm{X} \rightarrow \mathrm{D}(\mathrm{T})$, given by

$$
\mathrm{J}_{\lambda} x=x-\lambda \mathrm{J}^{-1}\left(\mathrm{~T}_{\lambda} x\right),
$$

are called the Yosida "approximant" and "resolvent" of $T$, respectively. The operator $T_{\lambda}$ is bounded, continuous and monotone (i.e., maximal monotone) and the operator $\mathrm{J}_{\lambda}$ is bounded and continuous. It is also known that $T_{\lambda} x \in T\left(J_{\lambda} x\right)$ for every $x \in X$ and $\lim _{\lambda \rightarrow 0} J_{\lambda} x=x$ for all $x \in \overline{c o D}(T)$, where $\operatorname{coD}(T)$ is the convex hull of $D(T)$. In addition, $T_{\lambda} x \rightarrow T^{(0)} x$ as $\lambda \rightarrow 0^{+}$for every $x \in D(T)$ where

$$
\left\|T^{0} x\right\|=\inf \left\{\left\|y^{*}\right\|: y^{*} \in T x\right\},
$$

and $\left\|T_{\lambda} x\right\| \leqslant|T x|$ for all $\lambda>0$ and $x \in D(T)$, where $|T x|$ denotes $\left\|T^{0} x\right\|$. The basic theory on monotone type operators can be found in Pascali and Sburlan [30], Barbu [9], Browder [13], Browder and Hess [17], Zeidler [34] and the references therein.

Lemma 1.2 is useful in the squeal. Its proof can be found in the proof of Theorem 2.7 due to Asfaw [6].

Lemma 1.2. Let $\mathrm{A}: \mathrm{X} \supseteq \mathrm{D}(\mathrm{A}) \rightarrow 2^{\mathrm{X}^{*}}$ be a maximal monotone operator. Let $\varepsilon>0, \mathrm{~A}_{\varepsilon}$ and $\mathrm{J}_{\varepsilon}^{\mathrm{A}}$ be the Yosida approximant and resolvent of $A$, respectively. If the sequences $\left\{x_{n}\right\}$ in $X$ and $\left\{\varepsilon_{n}\right\}$ in $(0, \infty)$, are bounded, then $\left\{\mathrm{J}_{\mathcal{E}_{\mathrm{n}}}^{\mathrm{A}} \mathrm{x}_{\mathrm{n}}\right\}$ is bounded.

For the readers' convenience we shall briefly outline the relevant degree theories for monotone type operators in reflexive Banach spaces. Let $T: X \supseteq D(T) \rightarrow 2^{X^{*}}$ and $A: X \supseteq D(A) \rightarrow 2^{X^{*}}$ be maximal monotone operators, $S: X \rightarrow 2^{X^{*}}$ be a bounded pseudomonotone operator and, $f: \bar{G} \rightarrow X^{*}$ and $g: \bar{G} \rightarrow X^{*}$ be bounded demicontinuous operators of type $\left(S_{+}\right)$.

Browder's degree theory (c.f., [16]) is for operators of $T+f$ with $0 \in T(0)$. It is useful to indicate that the definition of the Browder degree doesn't require that $T$ is densely defined. However, Kobayashi and Otani [22, Proposition 3.1] proved that the homotopy $\{(1-t)(T+f)+t g\}_{t \in[0,1]}$ is a pseudomonotone homotopy (c.f., [16]) if and only if $\mathrm{T}$ is densely defined. Hu and papageorgiou [18] generalized the Browder degree theory for everywhere defined, multi-valued and compact perturbation of $T+f$. Recently, Asfaw and Kartsatos [7] developed a degree theory for operators of $T+S$. The reader can find degree theories for perturbations of maximal monotone operators by a variant form of $\left(S_{+}\right)$operators in the papers by Berkovits and Mustonnen [10], Kartsatos and Skrypnik [19] and the references therein. In series of papers [3-5], Asfaw developed three degree theories for operators of the type (i) $T+A+S$ such that $A$ is of type 
$\Gamma_{\phi}$, (ii) $\mathrm{T}+\mathrm{S}+\mathrm{C}$ where $\mathrm{C}: \mathrm{X} \supseteq \mathrm{D}(\mathrm{C}) \rightarrow \mathrm{X}^{*}$ is a sublinear compact operator with $\mathrm{D}(\mathrm{T}) \subseteq \mathrm{D}(\mathrm{C})$ and $\mathrm{S}$ is bounded of type $\left(S_{+}\right)$, and (iii) $L+A+C$ where $L$ is possibly nonmonotone, linear, closed, densely defined and inverse compact, $A$ is of type $\Gamma_{\phi}$ and $C: X \rightarrow X^{*}$ is a bounded and demicontinuous operator. In [4] the conditions on $L$ allowed the operator $C$ to be demicontinuous (weaker than continuity). The possibility of $L$ to be nonmonotone restricted the usage of quasibounded assumption on $A$. All of these theories (except the case of (iii) due to Asfaw [4]) required perturbations of a single maximal monotone operator (or the sum of two maximal monotone operators) by single-valued or multi-valued operators of type $\left(S_{+}\right)$either densely defined or defined on a closure of a bounded and open subset of $X$. In contrast to these, it is the main goal of the present paper to develop degree theories for operators of the type $T+A+C$ where $T$ is of compact resolvent, $A$ is strongly quasibounded and $C: X \supseteq D(C) \rightarrow X^{*}$ is a bounded and continuous operator such that $D(A) \subseteq D(C)$ or $D(C)$ is the closure of a nonempty and open (possibly unbounded) subset of $X$. As a result the theories gave improvements of the theory in [4] by replacing the linear (possibly nonmonotone) operator $L$ by a resolvent compact (linear or nonlinear) maximal monotone operator $T$ such that $A$ is strongly quiasibounded and $C$ is bounded and continuous. These degree theories are new in the sense that the operator $C$ is not necessarily pseudomonotone type (e.g., not necessarily of type $\left(S_{+}\right)$) and $D(C)$ is neither dense nor equal to the closure of a bounded and open subset of $X$. These theories will help to derive applicable existence theorems which are not known earlier.

The arguments of the construction of these degrees are based on Leray-Scahuder degree along with Nagumo homotopy. The essential feature of Nagumo homotopy is that the Leray-Schauder degree $d_{L S}$ is invariant under the homotopy $\mathrm{H}:[0,1] \times \overline{\mathrm{G}} \rightarrow \mathrm{X}^{*}$ given by

$$
H(x, t)=x-K(t, x),(t, x) \in[0,1] \times \bar{G},
$$

provided that $G$ is a nonempty, bounded and open subset of $X, K:[0,1] \times \bar{G} \rightarrow X$ is a compact operator and $0 \notin H(t, \partial G)$ for all $t \in[0,1]$. Throughout the paper we shall use $d_{N A}$ instead of $d_{L S}$ whenever we use such homotopy. Additional details of Nagumo degree and related results can be found in Theorems 5, 6 and 7 due to Nagumo [28]. It is worth mentioning that the Leray-Schauder degree and Nagumo degree coincide in Banach space setting. In addition, the Leray-Schauder homotopy requires the mapping $x \mapsto K(t, x)$ to be compact for each $t \in[0,1]$ and $t \mapsto K(t, x)$ is uniformly continuous uniformly for all $x \in \overline{\mathrm{G}}$.

The paper is organized as follows. In Section 2 a degree theory for operators of the type $T+A+C$ with $\mathrm{D}(A) \subseteq \mathrm{D}(\mathrm{C})$, is developed. A homotopy invariance result along with basic properties are derived. Section 3 deals about a degree theory for operators of the form $T+A+C$ with $D(C)=\bar{U}$, where $U$ is a nonempty and open (possibly unbounded) subset of $X$. In Section 4 we applied the degree theories developed in Sections 2 and 3, and derived existence theorems concerning solvability of inclusion (e.g., variational inequality) problems. The last section demonstrates the applicability of the theory for a nonlinear parabolic variational inequality problem. Throughout Sections 2 and 3 we shall use the symbols $d_{L S}$ and $d_{N A}$ to denote Leray-Schauder and Nagumo degrees, respectively. The letter $d$ stands for each of the degree mappings constructed in Sections 2 and 3.

\section{Degree theory for $T+A+C$ with $D(A) \subseteq D(C)$}

We are now ready to construct the required degree mapping for operators of the type $T+A+C$ with $\mathrm{D}(\mathrm{A}) \subseteq \mathrm{D}(\mathrm{C})$. Theorem 2.1 is used in the squeal. The proof of Theorem 2.1 used the Leray-Schauder degree and Nagumo homotopy.

Theorem 2.1. Let $\mathrm{G}$ be a nonempty, bounded and open subset of $\mathrm{X}$. Let $\mathrm{T}: \mathrm{X} \supseteq \mathrm{D}(\mathrm{T}) \rightarrow 2^{\mathrm{X}^{*}}$ with $0 \in \mathrm{T}(0)$ and $\mathrm{A}: \mathrm{X} \supseteq \mathrm{D}(\mathrm{A}) \rightarrow 2^{\mathrm{X}^{*}}$ be maximal monotone operators such that $\mathrm{T}$ is of compact resolvent, $\mathrm{A}$ is strongly quasibounded and, $\mathrm{C}: \mathrm{X} \supseteq \mathrm{D}(\mathrm{C}) \rightarrow \mathrm{X}^{*}$ and $\mathrm{B}: \overline{\mathrm{G}} \rightarrow \mathrm{X}^{*}$ be bounded and continuous operators such that $\langle\mathrm{B} x, x\rangle \geqslant 0$ for all $x \in \mathrm{G},\langle\mathrm{B} x, x\rangle>0$ for all $x \in \partial \mathrm{G}$ and $\mathrm{D}(\mathrm{A}) \subseteq \mathrm{D}(\mathrm{C})$. Let

$$
H(t, x)=T x+t\left(A x+C x-f^{*}\right)+(1-t) B x,
$$


for $(\mathrm{t}, \mathrm{x}) \in[0,1] \times(\mathrm{D}(\mathrm{T}) \cap \mathrm{D}(\mathrm{A}) \cap \overline{\mathrm{G}})$. Assume that

$$
0 \notin H(t, D(T) \cap D(A) \cap \partial G),
$$

for all $\mathrm{t} \in[0,1]$. Then

$$
\left.d_{N A}\left(I-(T+J)^{-1}\left(-t\left(A_{\varepsilon}+C J_{\varepsilon}^{A}-f^{*}\right)\right)-(1-t) B+J\right), G, 0\right),
$$

is well-defined and, constant for all $\mathrm{t} \in[0,1]$ and sufficiently small $\varepsilon>0$.

Proof. We shall first show that

$$
d_{N A}\left(I-(T+J)^{-1}\left(-t\left(A_{\varepsilon}+C J_{\varepsilon}^{A}-f^{*}\right)-(1-t) B+J\right), G, 0\right),
$$

is well-defined for all $t \in[0,1]$ and sufficiently small $\varepsilon>0$, i.e., there exists $\varepsilon_{0}>0$ such that

$$
0 \notin\left(I-(T+J)^{-1}\left(-t\left(A_{\varepsilon}+C J_{\varepsilon}^{A}-f^{*}\right)-(1-t) B+J\right)(\partial G),\right.
$$

for all $\mathrm{t} \in[0,1]$ and $\varepsilon \in\left(0, \varepsilon_{0}\right)$. Suppose not, i.e., there exist $\varepsilon_{n} \downarrow 0^{+}, t_{n} \in[0,1]$ and $x_{n} \in \partial G$ such that

$$
x_{n}=(T+J)^{-1}\left(-t_{n}\left(A_{\varepsilon_{n}} x_{n}+C J_{\varepsilon_{n}}^{A} x_{n}-f^{*}\right)-\left(1-t_{n}\right) B x_{n}+J x_{n}\right),
$$

for all $n$, i.e., $x_{n} \in D(T) \cap \partial G$ for all $n$ and there exists $z_{n}^{*} \in T x_{n}$ such that

$$
\left.z_{n}^{*}+t_{n}\left(A_{\varepsilon_{n}} x_{n}+C\right)_{\varepsilon_{n}}^{A} x_{n}-f^{*}\right)+\left(1-t_{n}\right) B x_{n}=0,
$$

for all $n$. The boundedness of $\left\{x_{n}\right\}$ along with Lemma 1.2 imply the boundedness of $\left\{J_{\varepsilon_{n}}^{A} x_{n}\right\}$. In addition $\left\{C J_{\mathcal{E}_{n}}^{A} x_{n}\right\}$ and $\left\{B x_{n}\right\}$ are bounded because the operators $C$ and $B$ are bounded. Clearly $t_{n} \neq 0$ for all $n$. Otherwise, if $t_{n}=0$ for some $n$, we get $z_{n}^{*}+B x_{n}=0$ with $x_{n} \in D(T) \cap \partial G$, i.e., the monotonicity of $T$ with $0 \in \mathrm{T}(0)$ and condition of $\mathrm{B}$ on $\partial \mathrm{G}$ imply

$$
0<\left\langle B x_{n}, x_{n}\right\rangle=-\left\langle z_{n}^{*}, x_{n}\right\rangle \leqslant 0,
$$

for all $n$, i.e., $0<0$, which is absurd. This shows that $t_{n} \neq 0$ for all $n$. The monotonicity of $T$ with $0 \in T(0)$ and $\langle\mathrm{B} x, \mathrm{x}\rangle>0$ for all $x \in \partial \mathrm{G}$ imply that

$$
\begin{aligned}
\left\langle A_{\varepsilon_{n}} x_{n}, x_{n}\right\rangle= & -\frac{1}{t_{n}}\left\langle z_{n}^{*}, x_{n}\right\rangle-\frac{1-t_{n}}{t_{n}}\left\langle B x_{n}, x_{n}\right\rangle \\
& -\left\langle C J_{\varepsilon_{n}}^{A} x_{n}-f^{*}, x_{n}\right\rangle \leqslant K,
\end{aligned}
$$

for all $n$, where $K$ is an upper bound for $\left\{-\left\langle C_{\varepsilon_{n}} J_{\varepsilon_{n}}^{A} x_{n}-f^{*}, x_{n}\right\rangle\right\}$. Due to the properties of $A_{\varepsilon_{n}}$ and $J_{\varepsilon_{n}}^{A}$ it follows that $J_{\mathcal{E}_{n}}^{A} x_{n} \in D(A), A_{\varepsilon_{n}} x_{n} \in A\left(J_{\varepsilon_{n}}^{A} x_{n}\right)$ and

$$
\mathrm{J}_{\varepsilon_{n}}^{A} x_{n}=x_{n}-\varepsilon_{n} J^{-1}\left(A_{\varepsilon_{n}} x_{n}\right),
$$

for all $n$, i.e., we get

$$
\begin{aligned}
\left\langle A_{\varepsilon_{n}} x_{n}, J_{\varepsilon_{n}}^{A} x_{n}\right\rangle & =\left\langle A_{\varepsilon_{n}} x_{n}, x_{n}-\varepsilon_{n} J^{-1}\left(A_{\varepsilon_{n}} x_{n}\right)\right\rangle \\
& =\left\langle A_{\varepsilon_{n}} x_{n}, x_{n}\right\rangle-\varepsilon_{n}\left\langle A_{\varepsilon_{n}} x_{n}, J^{-1}\left(A_{\varepsilon_{n}} x_{n}\right)\right\rangle \\
& =\left\langle A_{\varepsilon_{n}} x_{n}, x_{n}\right\rangle-\varepsilon_{n}\left\|A_{\varepsilon_{n}} x_{n}\right\|^{2} \\
& \leqslant\left\langle A_{\varepsilon_{n}} x_{n}, x_{n}\right\rangle \leqslant K
\end{aligned}
$$

for all $n$. The strong quasiboundedness of $A$ shows the boundedness of $\left\{A_{\varepsilon_{n}} x_{n}\right\}$, i.e., (2.1) implies that $\left\{z_{\mathrm{n}}^{*}\right\}$ is bounded, and

$$
z_{n}^{*}+J x_{n}=-t_{n}\left(A_{\varepsilon_{n}} x_{n}+C J_{\varepsilon_{n}}^{A} x_{n}-f^{*}\right)-\left(1-t_{n}\right) B x_{n}+J x_{n}=E_{n}
$$


for all $n$. The compactness of $(T+J)^{-1}$ and boundedness of $\left\{E_{n}\right\}$ imply that there exists a subsequence of $\left\{x_{n}\right\}$, denoted again by $\left\{x_{n}\right\}$, such that

$$
x_{n}=(T+J)^{-1}\left(E_{n}\right) \rightarrow x_{0} \in \partial G, \quad \text { and } \quad J_{\varepsilon_{n}}^{A} x_{n}=x_{n}-\varepsilon_{n} J^{-1}\left(A_{\varepsilon_{n}} x_{n}\right) \rightarrow x_{0},
$$

as $n \rightarrow \infty$. Assume without loss of generality that $z_{n}^{*} \rightarrow z_{0}^{*}, A_{\varepsilon_{n}} x_{n} \rightarrow a_{0}^{*}$ and $t_{n} \rightarrow t_{0}$ as $n \rightarrow \infty$. The maximal monotonicity of $T$ and $A$ implies that $G(T)$ and $G(A)$ are strong-weak closed in $X \times X^{*}$ (c.f., Brézis et al. [11]), i.e., $\left(x_{0}, z_{0}^{*}\right) \in G(T)$ and $\left(x_{0}, a_{0}^{*}\right) \in G(A)$, i.e., we get

$$
x_{0} \in \mathrm{D}(\mathrm{T}) \cap \mathrm{D}(\mathrm{A}) \cap \partial \mathrm{G} \subseteq \mathrm{D}(\mathrm{C}), \quad z_{0}^{*} \in \mathrm{T} x_{0} \quad \text { and } \quad \mathrm{a}_{0}^{*} \in A x_{0} .
$$

The continuity of $C$ and $B$ imply $C J_{\mathcal{E}_{n}}^{A} x_{n} \rightarrow C x_{0}$ and $B x_{n} \rightarrow B x_{0}$ as $n \rightarrow \infty$. Letting $n \rightarrow \infty$ in (2.1) gives

$$
0 \in\left(T+t_{0}\left(A+C-f^{*}\right)+\left(1-t_{0}\right) B\right)(D(T) \cap D(A) \cap \partial G) .
$$

But this is impossible, i.e., the claim holds.

Next we show that

$$
d_{N A}\left(I-(T+J)^{-1}\left(-t\left(A_{\varepsilon}+C J_{\varepsilon}^{A}-f^{*}\right)-(1-t) B+J\right), G, 0\right),
$$

is independent of $t \in[0,1]$ and $\varepsilon \in\left(0, \varepsilon_{0}\right)$. Fix $\varepsilon \in\left(0, \varepsilon_{0}\right)$ temporarily. Let $H_{\varepsilon}:[0,1] \times \bar{G} \rightarrow X^{*}$ be given by

$$
H_{\varepsilon}(t, x)=(T+J)^{-1}\left(-t\left(A_{\varepsilon} x+C J_{\varepsilon}^{A} x-f^{*}\right)-(1-t) B x+J x\right) .
$$

It is clear to see that $H_{\varepsilon}$ is a compact operator such that $0 \notin \mathrm{H}_{\varepsilon}(t, \partial G)$ for all $t \in[0,1]$, i.e., $\left\{H_{\varepsilon}\right\}_{t \in[0,1]}$ is an admissible Nagumo homotopy, i.e., we obtain that

$$
d_{N A}\left(I-H_{\varepsilon}(t, .), G, 0\right),
$$

is independent of $t \in[0,1]$, i.e., it follows that

$$
\begin{aligned}
d_{N A}\left(I-H_{\varepsilon}(t, .), G, 0\right) & =d_{L S}\left(I-H_{\mathcal{E}}(0, .), G, 0\right) \\
& =d_{L S}\left(I-(T+J)^{-1}(-B+J), G, 0\right),
\end{aligned}
$$

for all $t \in[0,1]$. Since $d_{L S}\left(I-(T+J)^{-1}(-B+J), G, 0\right)$ is independent of $\varepsilon \in\left(0, \varepsilon_{0}\right)$, we conclude that

$$
d_{N A}\left(I-H_{\varepsilon}(t, .), G, 0\right) \text {, }
$$

is independent of $t \in[0,1]$ and $\varepsilon \in\left(0, \varepsilon_{0}\right)$. This completes the proof.

Next we give the definition of the degree mapping.

Definition 2.2. Let $G$ be a nonempty, bounded and open subset of $X$. Let $T: X \supseteq D(T) \rightarrow 2^{X^{*}}$ and $A: X \supseteq D(A) \rightarrow 2^{X^{*}}$ be maximal monotone operators such that $T$ is resolvent compact with $0 \in \mathrm{T}(0)$, $A$ is strongly quasibounded and $C: X \supseteq D(C) \rightarrow X^{*}$ be a bounded and continuous operator with $\mathrm{D}(\mathrm{A}) \subseteq \mathrm{D}(\mathrm{C})$. Suppose

$$
f^{*} \notin(T+A+C)(D(T) \cap D(A) \cap \partial G) .
$$

Then the degree of $T+A+C$ at $f^{*}$ with respect to $G$, denoted by $d$, is defined by

$$
d\left(T+A+C, G, f^{*}\right)=\lim _{\varepsilon \downarrow 0^{+}} d_{L S}\left(I-(T+J)^{-1}\left(-A_{\varepsilon}-C J_{\varepsilon}^{A}+f^{*}+J\right), G, 0\right) .
$$

The basic properties of $\mathrm{d}$ are presented below.

Theorem 2.3. Let $\mathrm{G}$ be a bounded and open subset of $\mathrm{X}$. Let $\mathrm{T}: \mathrm{X} \supseteq \mathrm{D}(\mathrm{T}) \rightarrow 2^{\mathrm{X}^{*}}$ and $\mathrm{A}: \mathrm{X} \supseteq \mathrm{D}(\mathrm{A}) \rightarrow 2^{\mathrm{X}^{*}}$ be maximal monotone operators such that $\mathrm{T}$ is resolvent compact with $0 \in \mathrm{T}(0), \mathrm{A}$ is strongly quasibounded and $\mathrm{C}: \mathrm{X} \supseteq \mathrm{D}(\mathrm{C}) \rightarrow \mathrm{X}^{*}$ be a bounded and continuous operator with $\mathrm{D}(\mathrm{A}) \subseteq \mathrm{D}(\mathrm{C})$. Then the following hold. 
(i) (Normalization) $\mathrm{d}(\mathrm{T}+\mathrm{J}, \mathrm{G}, 0)=1$ if $0 \in \mathrm{G}$ and $\mathrm{d}(\mathrm{T}+\mathrm{J}, \mathrm{G}, 0)=0$ if $0 \notin \overline{\mathrm{G}}$.

(ii) (Existence) Suppose

$$
f^{*} \notin(T+A+C)(D(T) \cap D(A) \cap \partial G) \text { and } d\left(T+A+C, G, f^{*}\right) \neq 0 .
$$

Then $f^{*} \in(T+A+C)(D(T) \cap D(A) \cap G)$.

(iii) (Translation Invariance) Let $\mathrm{f}^{*} \notin(T+A+C)(D(T) \cap D(A) \cap \partial G)$. Then we have

$$
d\left(T+A+C-f^{*}, G, 0\right)=d\left(T+A+C, G, f^{*}\right) .
$$

(iv) (Decomposition) Let $\mathrm{G}_{1}$ and $\mathrm{G}_{2}$ be nonempty and disjoint open subsets of $\mathrm{G}$ such that

$$
f^{*} \notin(T+A+C)\left(D(T) \cap D(A) \cap\left(\bar{G} \backslash\left(G_{1} \cup G_{2}\right)\right)\right) .
$$

Then

$$
d\left(T+A+C, G, f^{*}\right)=d\left(T+A+C, G_{1}, f^{*}\right)+d\left(T+A+C, G_{2}, f^{*}\right) .
$$

(v) Let $\mathrm{B}: \overline{\mathrm{G}} \rightarrow \mathrm{X}^{*}$ be a bounded and continuous operator such that $\langle\mathrm{B} x, \mathrm{x}\rangle>0$ for all $\mathrm{x} \in \partial \mathrm{G}$ and $\langle\mathrm{Bx}, \mathrm{x}\rangle \geqslant 0$ for all $\mathrm{x} \in \overline{\mathrm{G}}$ and

$$
H:[0,1] \times(D(T) \cap D(A) \cap \bar{G}) \rightarrow 2^{X^{*}},
$$

be given by

$$
H(t, x)=T x+t\left(A x+C x-f^{*}\right)+(1-t) B x .
$$

Suppose $0 \notin \mathrm{H}(\mathrm{t}, \mathrm{D}(\mathrm{T}) \cap \mathrm{D}(\mathrm{A}) \cap \partial \mathrm{G})$ for all $\mathrm{t} \in[0,1]$. Then $\mathrm{d}\left(\mathrm{H}(\mathrm{t},),. \mathrm{G}, \mathrm{f}^{*}\right)$ is independent of $\mathrm{t} \in[0,1]$.

Proof.

(i) By the definition of $d$ and using $(T+J)(0) \ni 0\left((T+J)^{-1}(0)=0\right)$, it follows that

$$
\begin{aligned}
\mathrm{d}(\mathrm{T}+\mathrm{J}, \mathrm{G}, 0) & \left.=\mathrm{d}_{\mathrm{LS}}\left(\mathrm{I}-(\mathrm{T}+\mathrm{J})^{-1}(-\mathrm{J}+\mathrm{J})\right), \mathrm{G}, 0\right) \\
& =\mathrm{d}_{\mathrm{LS}}(\mathrm{I}, \mathrm{G}, 0) .
\end{aligned}
$$

Clearly $d_{L S}(I, G, 0)=1$ if $0 \in G$ and $d_{L S}(I, G, 0)=0$ if $0 \notin \bar{G}$. The proof of (i) follows.

(ii) Assume that $f^{*} \notin(T+A+C)(D(T) \cap D(A) \cap \partial G)$ and $d\left(T+A+C, G, f^{*}\right) \neq 0$. Then

$$
d_{L S}\left(I-(T+J)^{-1}\left(-A_{\varepsilon}-C J_{\varepsilon}^{A}+f^{*}+J\right), G, 0\right) \neq 0,
$$

for all sufficiently small $\varepsilon>0$, i.e., for each $\varepsilon_{n} \downarrow 0^{+}$there exists $x_{n} \in D(T) \cap G$ and $z_{\mathfrak{n}}^{*} \in T x_{n}$ such that

$$
z_{n}^{*}+A_{\varepsilon_{n}} x_{n}+C J_{\mathcal{E}_{n}}^{A} x_{n}=f^{*},
$$

for all $\mathrm{n}$. The arguments in the proof of Theorem 2.1 can be applied to conclude that

$$
f^{*} \in(T+A+C)(D(T) \cap D(A) \cap G) .
$$

The details are omitted here.

(iii) Suppose $f^{*} \notin(T+A+C)(D(T) \cap D(A) \cap \partial G)$. The definition of $d$ gives

$$
\begin{aligned}
d\left(T+A+C-f^{*}, G, 0\right) & =d_{L S}\left(I-(T+J)^{-1}\left(-A_{\varepsilon}-C J_{\varepsilon}^{A}+f^{*}+J\right), G, 0\right) \\
& =d\left(T+A+C, G, f^{*}\right),
\end{aligned}
$$

for all sufficiently small $\varepsilon>0$, i.e., translation invariance follows. 
(iv) By the decomposition property of Leray-Schauder degree, we see that

$$
\begin{aligned}
d\left(T+A+C, G, f^{*}\right)= & d_{L S}\left(I-(T+J)^{-1}\left(-A_{\varepsilon}-C J_{\varepsilon}^{A}+f^{*}+J\right), G, 0\right) \\
= & d_{L S}\left(I-(T+J)^{-1}\left(-A_{\varepsilon}-C J_{\varepsilon}^{A}+f^{*}+J\right), G_{1}, 0\right) \\
& +d_{L S}\left(I-(T+J)^{-1}\left(-A_{\varepsilon}-C J_{\varepsilon}^{A}+f^{*}+J\right), G_{2}, 0\right),
\end{aligned}
$$

for all sufficiently small $\varepsilon>0$. Letting $\varepsilon \downarrow 0^{+}$gives

$$
\begin{aligned}
d\left(T+A+C, G, f^{*}\right) & =d\left(T+A+C-f^{*}, G_{1}, 0\right)+d\left(T+A+C-f^{*}, G_{2}, 0\right) \\
& =d\left(T+A+C, G_{1}, f^{*}\right)+d\left(T+A+C, G_{2}, f^{*}\right),
\end{aligned}
$$

(v) The proof is given in Theorem 2.1.

\section{Degree theory for $T+A+C$ with $D(C)=\bar{U}$}

Next we construct our degree mapping for operators of the type $T+A+C$ with $D(C)=\bar{U}$ where $U$ is a nonempty and open subset of $X$. We shall complete the construction based on Theorem 3.1. The proof requires the regularity property of a topological space.

Theorem 3.1. Let $\mathrm{U}$ be a nonempty and open subset of $\mathrm{X}, \mathrm{T}: \mathrm{X} \supseteq \mathrm{D}(\mathrm{T}) \rightarrow 2^{\mathrm{X}^{*}}$ and $\mathrm{A}: \mathrm{X} \supseteq \mathrm{D}(\mathrm{A}) \rightarrow 2^{\mathrm{X}^{*}}$ be maximal monotone operators such that $\mathrm{T}$ is of compact resolvents, $\mathrm{A}$ is strongly quasibounded and $\mathrm{C}: \overline{\mathrm{U}} \rightarrow \mathrm{X}^{*}$ and $\mathrm{B}: \mathrm{X} \rightarrow \mathrm{X}^{*}$ are bounded and continuous operators such that $\langle\mathrm{Bx}, \mathrm{x}\rangle>0$ for all $\mathrm{x} \in \mathrm{X} \backslash\{0\}$ and $\langle\mathrm{B} x, \mathrm{x}\rangle \geqslant 0$ for all $x \in X$. Let $\mathrm{S}:[0,1] \times(\mathrm{D}(\mathrm{T}) \cap \mathrm{D}(\mathrm{A}) \cap \overline{\mathrm{U}}) \rightarrow 2^{\mathrm{X}^{*}}$ be given

$$
S(t, x)=T x+t\left(A x+C x-f^{*}\right)+(1-t) B x .
$$

Suppose $0 \notin \mathrm{S}(\mathrm{t}, \mathrm{D}(\mathrm{T}) \cap \mathrm{D}(\mathrm{A}) \cap \partial \mathrm{U})$ for all $\mathrm{t} \in[0,1]$. Then

(i) there exist a nonempty, bounded and open subset $\mathrm{G}$ of $\mathrm{X}$ such that $\overline{\mathrm{G}} \subseteq \mathrm{U}$ and

$$
\mathrm{Z}\left(\mathrm{S}, \mathrm{f}^{*}\right)=\bigcup_{0 \leqslant t \leqslant 1}\{x \in \mathrm{D}(\mathrm{T}) \cap \mathrm{D}(\mathrm{A}) \cap \mathrm{U}: \mathrm{S}(\mathrm{t}, \mathrm{x}) \ni 0\} \subseteq \mathrm{G} .
$$

(ii)

$$
d_{N A}\left(I-(T+J)^{-1}\left(-t\left(A_{\varepsilon}+C J_{\varepsilon}^{A}-f^{*}\right)-(1-t) B+J\right), G, 0\right)
$$

is independent of $\mathrm{t} \in[0,1], \mathrm{G}$ and sufficiently small $\varepsilon>0$.

Proof.

(i) We shall show that $Z\left(S, f^{*}\right)$ is a compact subset of $U$. To this end, let $\left\{x_{n}\right\}$ be a bounded sequence in $Z\left(S, f^{*}\right)$, i.e., $x_{n} \in D(T) \cap D(A) \cap U$ and there exits $t_{n} \in[0,1]$ such that

$$
0 \in T x_{n}+t_{n}\left(A x_{n}+C x_{n}-f^{*}\right)+\left(1-t_{n}\right) B x_{n},
$$

for all $n$, i.e., there exist $u_{n}^{*} \in T x_{n}$ and $w_{n}^{*} \in A x_{n}$ such that

$$
u_{n}^{*}+t_{n}\left(w_{n}^{*}+C x_{n}-f^{*}\right)+\left(1-t_{n}\right) B x_{n}=0, \text { for all } n \text {. }
$$

Clearly $\left\{x_{n}\right\},\left\{t_{n} C x_{n}\right\}$ and $\left\{\left(1-t_{n}\right) B x_{n}\right\}$ are bounded. The boundedness of $\left\{w_{n}^{*}\right\}$ follows because of the strong quasiboundedness of $A$ and following the arguments of the proof of Theorem 2.1. The compactness of $(\mathrm{T}+\mathrm{J})^{-1}$ and boundedness of

$$
\left\{D_{n}=-t_{n}\left(w_{n}^{*}+C x_{n}-f^{*}\right)-\left(1-t_{n}\right) B x_{n}+J x_{n}\right\},
$$


imply the existence of a subsequence, denoted again by $\left\{x_{n}\right\}$, such that

$$
x_{n}=(T+J)^{-1}\left(D_{n}\right) \rightarrow x_{0} \in \overline{\mathrm{U}}, \text { as } \mathrm{n} \rightarrow \infty .
$$

The arguments of the proof of Theorem 2.1 can be applied to show that

$$
x_{0} \in \mathrm{D}(\mathrm{T}) \cap \mathrm{D}(\mathrm{A}) \cap \overline{\mathrm{u}}, \text { and } 0 \in \mathrm{S}\left(\mathrm{t}_{0}, \mathrm{x}_{0}\right),
$$

for some $t_{0} \in[0,1]$. The boundary condition gives $x_{0} \in D(T) \cap D(A) \cap U$, i.e., $x_{0} \in Z\left(S, f^{*}\right)$. This proves the compactness of $Z\left(S, f^{*}\right)$. The regularity property of the normed space $X$ gives the existence of a nonempty, bounded and open subset $\mathrm{G}$ of $\mathrm{U}$ such that $\mathrm{Z}\left(\mathrm{S}, \mathrm{f}^{*}\right) \subseteq \mathrm{G} \subseteq \overline{\mathrm{G}} \subseteq \mathrm{U}$.

(ii) Fix G temporarily. The arguments used in the proof of Theorem 2.1 can be applied to conclude that

$$
d_{N A}\left(I-K_{\varepsilon}(t, .), G, 0\right)=d_{L S}\left(I-(T+J)^{-1}(-B+J), G, 0\right),
$$

for all $t \in[0,1]$ and sufficiently small $\varepsilon>0$, where

$$
K_{\varepsilon}(t, x)=(T+J)^{-1}\left(-t\left(A_{\varepsilon} x+C J_{\varepsilon}^{A} x-f^{*}\right)-(1-t) B x+J x\right),
$$

for $(t, x) \in[0,1] \times \bar{G}$. It remains to show that

$$
d_{L S}\left(I-(T+J)^{-1}(-B+J), G, 0\right),
$$

is independent of $G$, i.e.,

$$
d_{L S}\left(I-(T+J)^{-1}(-B+J), G_{1}, 0\right)=d_{L S}\left(I-(T+J)^{-1}(-B+J), G_{2}, 0\right),
$$

for any $G_{1}$ and $G_{2}$ satisfying $Z\left(S, f^{*}\right) \subseteq G_{1} \cap G_{2}$. Since $Z\left(S, f^{*}\right) \subseteq G_{1} \cap G_{2}$ and

$$
\overline{\mathrm{G}_{1} \backslash \overline{\mathrm{G}}_{2}} \subseteq \overline{\mathrm{G}}_{1} \backslash \mathrm{G}_{2}=\left(\mathrm{G}_{1} \backslash \mathrm{G}_{2}\right) \cup\left(\partial \mathrm{G}_{1} \backslash \mathrm{G}_{2}\right),
$$

it follows that

$$
0 \notin\left(I-(T+J)^{-1}(-B+J)\right)\left(\partial\left(G_{1} \cap G_{2}\right)\right),
$$

and

$$
0 \notin\left(I-(T+J)^{-1}(-B+J)\right)\left(\partial\left(G_{1} \backslash \bar{G}_{2}\right)\right)
$$

for all $t \in[0,1]$. Let

$$
\Omega=\overline{\mathrm{G}}_{1} \backslash\left(\left(\mathrm{G}_{1} \cap \mathrm{G}_{2}\right) \cup\left(\mathrm{G}_{1} \backslash \overline{\mathrm{G}}_{2}\right)\right) .
$$

It is not hard to see that $G_{1} \cap \bar{G}_{2}$ and $G_{1} \backslash \bar{G}_{2}$ are nonempty, disjoint and open subsets of $G_{1}$,

$$
\partial\left(\mathrm{G}_{1} \backslash \overline{\mathrm{G}}_{2}\right) \cap\left(\mathrm{G}_{1} \cap \mathrm{G}_{2}\right)=\emptyset,
$$

and

$$
0 \notin\left(I-(T+J)^{-1}(-B+J)\right)\left(G_{1} \backslash \bar{G}_{2}\right) .
$$

By the decomposition property of Leray-Schauder degree, we obtain that

$$
\begin{aligned}
\mathrm{d}_{\mathrm{LS}}\left(\mathrm{I}-(\mathrm{T}+\mathrm{J})^{-1}(-\mathrm{B}+\mathrm{J}), \mathrm{G}_{1}, 0\right)= & \mathrm{d}_{\mathrm{LS}}\left(\mathrm{I}-(\mathrm{T}+\mathrm{J})^{-1}(-\mathrm{B}+\mathrm{J}), \Omega_{1}, 0\right) \\
& +\mathrm{d}_{\mathrm{LS}}\left(\mathrm{I}-(\mathrm{T}+\mathrm{J})^{-1}(-\mathrm{B}+\mathrm{J}), \Omega_{2}, 0\right),
\end{aligned}
$$

where $\Omega_{1}=G_{1} \cap G_{2}$ and $\Omega_{2}=G_{1} \backslash \bar{G}_{2}$. On the other hand, one can apply the existence property of the Leray-Schauder degree to conclude

$$
d_{L S}\left(I-(T+J)^{-1}(-B+J), G_{1} \backslash \bar{G}_{2}, 0\right)=0,
$$


because $\overline{\mathrm{G}_{1} \backslash \overline{\mathrm{G}}_{2}} \subseteq \overline{\mathrm{G}}_{1} \backslash \mathrm{G}_{2}, \mathrm{Z}\left(\mathrm{S}, \mathrm{f}^{*}\right) \subseteq \mathrm{G}_{1} \cap \mathrm{G}_{2}$ and

$$
0 \notin\left(I-(T+J)^{-1}(-B+J)\right)\left(\overline{G_{1} \backslash \bar{G}_{2}}\right) .
$$

This gives

$$
d_{L S}\left(I-(T+J)^{-1}(-B+J), G_{1}, 0\right)=d_{L S}\left(I-(T+J)^{-1}(-B+J), \Omega_{1}, 0\right) .
$$

Analogously, we get

$$
d_{L S}\left(I-(T+J)^{-1}(-B+J), G_{2}, 0\right)=d_{L S}\left(I-(T+J)^{-1}(-B+J), \Omega_{1}, 0\right),
$$

i.e., we obtain

$$
d_{L S}\left(I-(T+J)^{-1}(-B+J), G_{1}, 0\right)=d_{L S}\left(I-(T+J)^{-1}(-B+J), G_{2}, 0\right) .
$$

The proof is completed.

Let

$$
Z\left(T+A+C, f^{*}\right)=\left\{x \in D(T) \cap D(A) \cap U: T x+A x+C x \ni f^{*}\right\} .
$$

The definition of the required degree mapping is given below.

Definition 3.2. Let $U$ be a nonempty and open subset of $X$. Let $T: X \supseteq D(T) \rightarrow 2^{X^{*}}$ and

$$
\mathrm{A}: \mathrm{X} \supseteq \mathrm{D}(\mathrm{A}) \rightarrow 2^{X^{*}},
$$

be maximal monotone operators such that $T$ is of compact resolvents with $0 \in T(0)$, $A$ is strongly quasibounded and $\mathrm{C}: \overline{\mathrm{U}} \rightarrow \mathrm{X}^{*}$ be a bounded and continuous operator. Suppose

$$
f^{*} \notin(T+A+C)(D(T) \cap D(A) \cap \partial U) .
$$

The degree of $T+A+C$ at $f^{*}$ with respect to $U$, denoted by $d\left(T+A+C, U, f^{*}\right)$, is defined by

$$
d\left(T+A+C, U, f^{*}\right)=\lim _{\varepsilon \downarrow 0^{+}} d_{L S}\left(I-(T+J)^{-1}\left(-A_{\varepsilon}-C J_{\varepsilon}^{A}+f^{*}+J\right), G, 0\right),
$$

where $G$ is a bounded and open subset of $U$ such that $Z\left(T+A+C, f^{*}\right) \subseteq G$ guaranteed by Theorem 3.1.

The degree mapping satisfies the following properties.

Theorem 3.3. Let $\mathrm{U}$ be a nonempty and open subset of $\mathrm{X}$. Let $\mathrm{T}: \mathrm{X} \supseteq \mathrm{D}(\mathrm{T}) \rightarrow 2^{\mathrm{X}^{*}}$ and $\mathrm{A}: \mathrm{X} \supseteq \mathrm{D}(\mathrm{A}) \rightarrow 2^{\mathrm{X}^{*}}$ be maximal monotone operators such that $\mathrm{T}$ is of compact resolvents with $0 \in \mathrm{T}(0), \mathrm{A}$ is strongly quasibounded and $\mathrm{C}: \overline{\mathrm{U}} \rightarrow \mathrm{X}^{*}$ be a bounded and continuous operator. Then the following hold:

(i) (Normalization) $\mathrm{d}(\mathrm{T}+\mathrm{J}, \mathrm{U}, 0)=1$ if $0 \in \mathrm{U}$, and $\mathrm{d}(\mathrm{T}+\mathrm{J}, \mathrm{U}, 0)=0$ if $0 \notin \overline{\mathrm{U}}$.

(ii) (Existence) If

$$
f^{*} \notin(T+A+C)(D(T) \cap D(A) \cap \partial U) \text {, and } d\left(T+A+C, U, f^{*}\right) \neq 0,
$$

then $\mathrm{f}^{*} \in(T+A+C)(D(T) \cap D(A) \cap U)$.

(iii) (Translation Invariance) Let $\mathrm{f}^{*} \notin(\mathrm{T}+\mathrm{A}+\mathrm{C})(\mathrm{D}(\mathrm{T}) \cap \mathrm{D}(\mathrm{A}) \cap \partial \mathrm{u})$. Then we have

$$
d\left(T+A+C, U, f^{*}\right)=d\left(T+A+C-f^{*}, u, 0\right) .
$$

(iv) (Decomposition) Let $\mathrm{U}_{1}$ and $\mathrm{U}_{2}$ be nonempty, disjoint and open subsets of $\mathrm{U}$ such that

$$
f^{*} \notin(T+A+C)\left(D(T) \cap D(A) \cap\left(\bar{U} \backslash\left(U_{1} \cup U_{2}\right)\right)\right),
$$

then

$$
d\left(T+A+C, U, f^{*}\right)=d\left(T+A+C, U_{1}, f^{*}\right)+d\left(T+A+C, U_{2}, f^{*}\right) .
$$


(v) (Homotopy invariance) Let $\mathrm{B}: \mathrm{X} \rightarrow \mathrm{X}^{*}$ be a bounded and continuous operator such that $\langle\mathrm{B} \mathrm{x}, \mathrm{x}\rangle>0$ for all $x \in X \backslash\{0\},\langle B x, x\rangle \geqslant 0$ for all $x \in X$ and

$$
H(t, x)=T x+t\left(A x+C x-f^{*}\right)+(1-t) B x,
$$

for $(t, x) \in[0,1] \times(D(T) \cap D(A) \cap \overline{\mathrm{U}})$, then $\mathrm{d}(\mathrm{H}(\mathrm{t},),. \mathrm{U}, 0)$ is independent of $\mathrm{t} \in[0,1]$ provided that $0 \notin \mathrm{H}(\mathrm{t}, \mathrm{D}(\mathrm{T}) \cap \mathrm{D}(\mathrm{A}) \cap \partial \mathrm{U})$ for all $\mathrm{t} \in[0,1]$.

Proof.

(i) We shall use $T, A=\{0\}, C=0$ and $B=J$ in Definition 3.2. Let $G$ be a nonempty, bounded and open subset of $\mathrm{U}$ guaranteed by (i) of Theorem 3.1. Then the definition of $d$ gives

$$
\mathrm{d}(\mathrm{T}+\mathrm{J}, \mathrm{U}, 0)=\mathrm{d}_{\mathrm{LS}}\left(\mathrm{I}-(\mathrm{T}+\mathrm{J})^{-1}(-\mathrm{J}+\mathrm{J}), \mathrm{G}, 0\right) .
$$

Suppose $0 \in U$. Since $0 \in T(0)\left((T+J)^{-1}(0)=0\right)$ (i.e., $\left.0 \in D(T) \cap U\right)$, it follows that $0 \in T x+J x$ is solvable with $x=0$ and $t=0$ (i.e., $0 \in \mathrm{G}$ ) and

$$
\mathrm{d}(\mathrm{T}+\mathrm{J}, \mathrm{U}, 0)=\mathrm{d}_{\mathrm{LS}}(\mathrm{I}, \mathrm{G}, 0)=1 .
$$

In addition, $0 \notin \overline{\mathrm{G}}$ if $0 \notin \overline{\mathrm{U}}$, i.e., we have $\mathrm{d}(\mathrm{T}+\mathrm{J}, \mathrm{U}, 0)=\mathrm{d}_{\mathrm{LS}}(\mathrm{I}, \mathrm{G}, 0)=0$.

(ii) Suppose $f^{*} \notin(T+A+C)(D(T) \cap D(A) \cap \partial U)$ and $d\left(T+A+C, U, f^{*}\right) \neq 0$. Let $G$ be a bounded and open subset of $X$ such that $Z\left(T+A+C, f^{*}\right) \subseteq G \subset \bar{G} \subseteq U$. Then by the definition of $d\left(T+A+C, U, f^{*}\right)$ we have

$$
d_{L S}\left(I-(T+J)^{-1}\left(-A_{\varepsilon}-C J_{\varepsilon}^{A}+f^{*}+J\right), G, 0\right) \neq 0,
$$

for all sufficiently small $\varepsilon>0$, i.e., for each $\varepsilon_{n} \downarrow 0^{+}$there exist $x_{n} \in D(T) \cap G$ and $z_{n}^{*} \in T x_{n}$ such that

$$
z_{n}^{*}+A_{\varepsilon_{n}} x_{n}+C J_{\varepsilon}^{A} x_{n}=f^{*},
$$

for all $\mathrm{n}$. The arguments of the proof of Theorem $2.1 \mathrm{imply}$

$$
f^{*} \in(T+A+C)(D(T) \cap D(A) \cap G) .
$$

The details are omitted here.

(iii) By the definition of $d$, we obtain that

$$
\begin{aligned}
d\left(T+A+C, U, f^{*}\right) & \left.=d_{L S}\left(I-(T+J)^{-1}\left(-A_{\varepsilon}-C\right)_{\varepsilon}^{A}+f^{*}+J\right), G, 0\right) \\
& =d\left(T+A+C-f^{*}, U, 0\right),
\end{aligned}
$$

for all sufficiently small $\varepsilon>0$ and $G$ is an open and bounded subset of $U$ such that $Z\left(T+A+C, f^{*}\right) \subseteq G$.

(iv) Suppose $U_{1}$ and $U_{2}$ are disjoint and open subsets of $U$ satisfying the boundary condition. It follows that

$$
f^{*} \notin(T+A+C)\left(D(T) \cap D(A) \cap \partial U_{1}\right),
$$

and

$$
f^{*} \notin(T+A+C)\left(D(T) \cap D(A) \cap \partial U_{2}\right) .
$$

We shall denote the restrictions of $C$ on $U_{1}$ and $U_{2}$ by the same letter $C$. Let $G_{1}$ and $G_{2}$ be bounded and open subsets of $\mathrm{U}_{1}$ and $\mathrm{U}_{2}$, respectively, such that

$$
\mathrm{Z}\left(\mathrm{T}+\mathrm{A}+\mathrm{C}, \mathrm{f}^{*}\right) \subseteq \mathrm{G}_{1} \subseteq \overline{\mathrm{G}}_{1} \subseteq \mathrm{U}_{1}
$$

and

$$
\mathrm{Z}\left(\mathrm{T}+\mathrm{A}+\mathrm{C}, \mathrm{f}^{*}\right) \subseteq \mathrm{G}_{2} \subseteq \overline{\mathrm{G}}_{2} \subseteq \mathrm{U}_{2}
$$


Clearly $G_{1}$ and $G_{2}$ are disjoint subsets of $G=G_{1} \cup G_{2}$. It is not difficult to see that

$$
0 \notin\left(I-(T+J)^{-1}\left(-A_{\varepsilon}-C J_{\varepsilon}^{A}+f^{*}+J\right)\right)\left(\partial G_{1}\right),
$$

and

$$
0 \notin\left(I-(T+J)^{-1}\left(-A_{\varepsilon}-C J_{\varepsilon}^{A}+f^{*}+J\right)\right)\left(\partial G_{2}\right),
$$

for all sufficiently small $\varepsilon>0$, and

$$
0 \notin\left(I-(T+J)^{-1}\left(-A_{\varepsilon}-C J_{\varepsilon}^{A}+f^{*}+J\right)\left(\bar{G} \backslash\left(G_{1} \cap G_{2}\right)\right),\right.
$$

for all sufficiently small $\varepsilon>0$. The decomposition property of Leary-Schauder degree mapping yields

$$
\begin{aligned}
d\left(T+A+C, U, f^{*}\right)= & d_{L S}\left(I-(T+J)^{-1}\left(-A_{\varepsilon}-C J_{\varepsilon}^{A}+f^{*}+J\right), G, 0\right) \\
= & d_{L S}\left(I-(T+J)^{-1}\left(-A_{\varepsilon}-C J_{\varepsilon}^{A}+f^{*}+J\right), G_{1}, 0\right) \\
& +d_{L S}\left(I-(T+J)^{-1}\left(-A_{\varepsilon}-C J_{\varepsilon}^{A}+f^{*}+J\right), G_{2}, 0\right),
\end{aligned}
$$

for all sufficiently small $\varepsilon>0$, i.e., letting $\varepsilon \rightarrow 0^{+}$, Definition 3.2 implies

$$
d\left(T+A+C, U, f^{*}\right)=d\left(T+A+C, U_{1}, f^{*}\right)+d\left(T+A+C, U_{2}, f^{*}\right) .
$$

This proves (iv). The proof of (v) is given in Theorem 3.1.

\section{Surjectivity and variational inequality theorems}

The degree theories developed in Sections 2 and 3 are applied to prove new existence theorems, i.e., Theorems 4.1 and 4.2 give surjectivity results under certain coercivity condition, while Theorem 4.5 yields a new result on solvability of variational inequality problems. It is important to mention that these theorems are new even if the operator $A$ is identically zero and $C$ is everywhere defined.

Theorem 4.1. Let $\mathrm{U}$ be a nonempty and open subset of $\mathrm{X}$ with $0 \in \mathrm{U}$. Let $\mathrm{T}: \mathrm{X} \supseteq \mathrm{D}(\mathrm{T}) \rightarrow 2^{\mathrm{X}^{*}}$ and

$$
\mathrm{A}: \mathrm{X} \supseteq \mathrm{D}(\mathrm{A}) \rightarrow 2^{\mathrm{X}^{*}},
$$

be maximal monotone operators such that $\mathrm{T}$ is of compact resolvents with $0 \in \mathrm{T}(0), \mathrm{A}$ is strongly quasibounded and $\mathrm{C}: \mathrm{X} \supseteq \mathrm{D}(\mathrm{C}) \rightarrow \mathrm{X}^{*}$ be a bounded and continuous operator. Let $\mathrm{f}^{*} \in \mathrm{X}^{*}$. Then

(i) $f^{*} \in(T+A+C)\left(D(T) \cap D(A) \cap B_{R}(0)\right)$ if $D(A) \subseteq D(C)$ and there exists $R>0$ such that

$$
\left\langle u^{*}+C x-f^{*}, x\right\rangle>0,
$$

for all $x \in \mathrm{D}(\mathrm{T}) \cap \mathrm{D}(\mathrm{A}) \cap \partial \mathrm{B}_{\mathrm{R}}(0)$ and $\mathrm{u}^{*} \in \mathrm{Ax}$.

(ii) $f^{*} \in(T+A+C)(D(T) \cap D(A) \cap U)$ if $D(C)=\bar{U}$ and

$$
\left\langle u^{*}+C x-f^{*}, x\right\rangle>0,
$$

for all $\mathrm{x} \in \mathrm{D}(\mathrm{T}) \cap \mathrm{D}(\mathrm{A}) \cap \partial \mathrm{u}$ and $\mathrm{u}^{*} \in A \mathrm{x}$.

Proof. The proofs of (i) and (ii) are similar. We shall give the proof of (ii). Consider the homotopy inclusion

$$
H(t, x)=T x+t\left(A x+C x-f^{*}\right)+(1-t) J x,
$$

for $(t, x) \in[0,1] \times(D(T) \cap D(A) \cap \bar{U})$. The boundary condition on $T+A+C$ gives

$$
\begin{aligned}
\left\langle z^{*}+t\left(u^{*}+C x-f^{*}\right)+(1-t) J x, x\right\rangle & =\left\langle z^{*}, x\right\rangle+t\left\langle u^{*}+C x-f^{*}, x\right\rangle+(1-t)\|x\|^{2} \\
& \geqslant t\left\langle u^{*}+C x-f^{*}, x\right\rangle+(1-t)\|x\|^{2} \\
& >0,
\end{aligned}
$$

for all $t \in[0,1], x \in D(T) \cap D(A) \cap \partial u, z^{*} \in T x$ and $u^{*} \in A x$, i.e., $0 \notin H(t, D(T) \cap D(A) \cap \partial u)$ for 
all $t \in[0,1]$. Thus the homotopy invariance property $((\mathrm{v})$ of Theorem 3.3 with $B=J)$ ensures that $d(H(t,), U, 0$.$) is independent of t \in[0,1]$. This means

$$
d(H(t, .), u, 0)=d\left(T+A+C, U, f^{*}\right)=d(T+J, u, 0)=1,
$$

for all $t \in[0,1]$. The existence property ((ii) of Theorem 3.3) shows that

$$
f^{*} \in(T+A+C)(D(T) \cap D(A) \cap U) .
$$

The proof of (i) follows using $G=B_{R}(0)$.

It is not difficult to see that the conditions in Theorem 4.1 give the solvability of the variational inequality problem $\operatorname{VIP}\left(T+A+C, K, f^{*}\right)$ if $0 \in \mathrm{D}(A) \cap \stackrel{\circ}{K}$ is assumed in (i) and (ii). Indeed, the condition $0 \in \mathrm{D}(A) \cap \stackrel{\circ}{\mathrm{K}}$ implies the strong quasiboundedness of $A+\partial \mathrm{I}_{K}$ because $A$ and $\partial \mathrm{I}_{K}$ are both strongly quasibounded, and (i) and (ii) imply analogous conditions for the operator $A+\partial \mathrm{I}_{K}$. Consequently, Theorem 4.1 yields $f^{*} \in R\left(T+A+\partial I_{K}+C\right)$, i.e., $\operatorname{VIP}\left(T+A+C, K, f^{*}\right)$ is solvable. In addition, the same conclusion holds if (ii) holds for $\mathrm{D}(\mathrm{C})=\mathrm{K}=\overline{\mathrm{U}}$ with $\mathrm{U}=\stackrel{\circ}{\mathrm{K}}$. In Theorem 4.2 below the strong quasiboundedness assumption on $A$ is omitted and $T$ is assumed to be quasibounded (not necessarily strongly quausibounded).

Theorem 4.2. Let $\mathrm{U}$ be a nonempty and open subset of $\mathrm{X}$. Let $\mathrm{T}: \mathrm{X} \supseteq \mathrm{D}(\mathrm{T}) \rightarrow 2^{\mathrm{X}^{*}}$ and $\mathrm{A}: \mathrm{X} \supseteq \mathrm{D}(\mathrm{A}) \rightarrow 2^{\mathrm{X}^{*}}$ be maximal monotone operators such that $\mathrm{T}$ is quasibounded and of compact resolvents and $\mathrm{C}: \mathrm{X} \supseteq \mathrm{D}(\mathrm{C}) \rightarrow \mathrm{X}^{*}$ be a bounded and continuous operator. Suppose $\mathrm{f}^{*} \in \mathrm{X}^{*}$ and $0 \in \mathrm{U} \cap \mathrm{T}(0) \cap \mathrm{A}(0)$. Then

(i) $f^{*} \in(T+A+C)\left(D(T) \cap D(A) \cap B_{R}(0)\right)$ if $D(A) \subseteq D(C)$ and there exists $R>0$ such that

$$
\left\langle C x-f^{*}, x\right\rangle>0,
$$

for all $x \in \mathrm{D}(\mathrm{A}) \cap \partial \mathrm{B}_{\mathrm{R}}(0)$.

(ii) $f^{*} \in(T+A+C)(D(T) \cap D(A) \cap U)$ if $D(C)=\bar{U}$ and

$$
\left\langle C x-f^{*}, x\right\rangle>0
$$

for all $x \in \partial U$.

Proof. Suppose (i) holds. Let $\varepsilon>0, A_{\varepsilon}$ and $\mathrm{J}_{\varepsilon}^{A}$ be the Yosida approximant and resolvent of $A$, respectively. Then it follows that $A_{\varepsilon}+C: D(C) \rightarrow X^{*}$ is a bounded and continuous operator. Since $0 \in T(0) \cap A(0)$ and, $T$ and $A$ are maximal monotone, it follows that $A_{\varepsilon}(0)=0$ and $\left\langle u^{*}, x\right\rangle \geqslant 0$ and $\left\langle A_{\varepsilon} x, x\right\rangle \geqslant 0$ for all $x \in \mathrm{D}(\mathrm{T})$ and $\mathrm{u}^{*} \in \mathrm{T} x$. Consequently, we see that

$$
\begin{aligned}
\left\langle u^{*}+t\left(A_{\varepsilon} x+C x-f^{*}\right)+(1-t) J x, x\right\rangle & =\left\langle u^{*}, x\right\rangle+t\left\langle C x-f^{*}, x\right\rangle+(1-t)\|x\|^{2} \\
& \geqslant t\left\langle C x-f^{*}, x\right\rangle+(1-t)\|x\|^{2}>0
\end{aligned}
$$

for all $t \in[0,1]$ and $x \in D(T) \cap D(A) \cap \partial B_{R}(0)$ and $u^{*} \in T x$, i.e., we have

$$
0 \notin\left(T+t\left(A_{\varepsilon}+C-f^{*}\right)+(1-t) J\right)\left(D(T) \cap D(A) \cap \partial B_{R}(0)\right),
$$

for all $t \in[0,1]$ and sufficiently small $\varepsilon>0$. The homotopy invariance and normalization properties of the degree given in Definition 2.2 imply

$$
d\left(T+A_{\varepsilon}+C, B_{R}(0), f^{*}\right)=d\left(T+J, B_{R}(0), 0\right)=1,
$$


for all sufficiently small $\varepsilon>0$, i.e., for each $\varepsilon_{n} \downarrow 0^{+}$there exist $x_{n} \in D(T) \cap B_{R}(0)$ and $z_{n}^{*} \in T x_{n}$ such that

$$
z_{n}^{*}+A_{\varepsilon_{n}} x_{n}+C x_{n}=f^{*},
$$

for all $n$. It is clear that $\left\{x_{n}\right\}$ and $\left\{C x_{n}\right\}$ are bounded. The boundedness of $\left\{z_{n}^{*}\right\}$ follows based on the quasiboundedness of $T$, i.e., $\left\{A_{\varepsilon_{n}} x_{n}\right\}$ is bounded. One can follow the arguments used in the proof of Theorem 2.1 to conclude that

$$
f^{*} \in(T+A+C)\left(D(T) \cap D(A) \cap B_{R}(0)\right) .
$$

The details are omitted here.

Suppose (ii) holds. Then as in the proof of (i) it follows that

$$
\left\langle u^{*}+t\left(A_{\varepsilon} x+C x-f^{*}\right)+(1-t) J x, x\right\rangle>0,
$$

for all $t \in[0,1]$ and $x \in D(T) \cap \partial U$ and $u^{*} \in T x$. The normalization and homotopy invariance of the degree constructed in Section 3 give

$$
d\left(T+A_{\varepsilon}+C, U, f^{*}\right)=d(T+J, U, 0)=1 .
$$

Let $G$ be a bounded and open subset of $U$ guaranteed by (i) of Theorem 3.1 such that $Z\left(T+A+C, f^{*}\right) \subseteq G$. It can be proved (as in the proof of Theorem 3.1) that

$$
\mathrm{Z}\left(\mathrm{T}+\mathrm{A}_{\varepsilon}+\mathrm{C}, \mathrm{f}^{*}\right) \subseteq \mathrm{G},
$$

for all sufficiently small $\varepsilon>0$, i.e., for each $\varepsilon_{n} \downarrow 0^{+}$there exist $x_{n} \in D(T) \cap G$ and $z_{n}^{*} \in T x_{n}$ such that

$$
z_{n}^{*}+A_{\varepsilon_{n}} x_{n}+C x_{n}=f^{*},
$$

for all $\mathrm{n}$. Analogous arguments can be applied to conclude that

$$
f^{*} \in(T+A+C)(D(T) \cap D(A) \cap G) .
$$

The proof is completed.

Corollary 4.3 gives a surjectivity result.

Corollary 4.3. Suppose hypotheses on $\mathrm{T}, \mathrm{A}$ and $\mathrm{C}$ are satisfied in Theorems 4.1 and 4.2 with $0 \in \mathrm{D}(\mathrm{A}) \subseteq \mathrm{D}(\mathrm{C})$, and (i) is replaced by coercivity of $\mathrm{C}$, i.e.,

$$
\inf _{x \in D(A)} \frac{\langle C x, x\rangle}{\|x\|} \rightarrow \infty, \quad \text { as }\|x\| \rightarrow \infty
$$

Then $\mathrm{T}+\mathrm{A}+\mathrm{C}$ is surjective.

Proof. Let $\mathrm{f}^{*} \in \mathrm{X}^{*}$. Choose $\mathrm{b}_{0}^{*} \in A(0)$. The monotonicity of $A$ and coercivity of $C$ imply

$$
\begin{aligned}
\inf _{x \in D(A), u^{*} \in A x} \frac{\left\langle u^{*}+C x-f^{*}, x\right\rangle}{\|x\|} & =\inf _{x \in D(A), u^{*} \in A x}\left(\frac{\left\langle u^{*}-b_{0}^{*}+C x, x\right\rangle}{\|x\|}+\frac{\left\langle b_{0}^{*}-f^{*}, x\right\rangle}{\|x\|}\right) \\
& \geqslant \inf _{x \in D(A)} \frac{\langle C x, x\rangle}{\|x\|}-\left(\left\|f^{*}\right\|+\left\|b_{0}^{*}\right\|\right) \rightarrow \infty,
\end{aligned}
$$

as $\|x\| \rightarrow \infty$, i.e., there exits $R=R\left(f^{*}\right)>0$ such that

$$
\left\langle u^{*}+C x-f^{*}, x\right\rangle>0,
$$

for all $x \in D(A) \cap \partial B_{R}(0)$. Then (i) of Theorem 4.1 is satisfied, i.e., we get

$$
f^{*} \in(T+A+C)\left(D(T) \cap D(A) \cap B_{R}(0)\right) .
$$

The surjectivity of $T+A+C$ follows because $f^{*} \in X^{*}$ is arbitrary. The proof under conditions of Theorem 4.2 follows analogously. 
Let $\phi: X \rightarrow(-\infty, \infty]$ be a proper, convex and lower semicontinuous function. The domain of $\phi$, denoted by $\mathrm{D}(\phi)$, is given by $\mathrm{D}(\phi)=\{x \in X: \phi(x)<\infty\}$. The subdifferential of $\phi$ (in the sense of convex analysis), denoted by $\partial \phi: \mathrm{X} \supseteq \mathrm{D}(\partial \phi) \rightarrow 2^{\mathrm{X}^{*}}$ is defined as

$$
\partial \phi(x)=\left\{u^{*} \in X^{*}:\left\langle u^{*}, x-y\right\rangle \geqslant \phi(x)-\phi(y), \forall y \in X\right\} .
$$

For a nonempty, closed and convex subset $K$ of $X$, let $I_{K}: X \rightarrow(-\infty, \infty]$ be the indicator function on $K$, i.e., $I_{K}(x)=0$ for all $x \in K$, and $I_{K}(x)=\infty$ for all $x \notin K$. It is known that $I_{K}$ is a proper, convex and lower semicontinuous function with domain K. Clearly $\left(x, w^{*}\right) \in G\left(\partial I_{K}\right)$ if $\left\langle w^{*}, x-y\right\rangle \geqslant 0$ for all $y \in K$.

Next recall Definition 4.4.

Definition 4.4. Let $K$ be a nonempty, closed and convex subset of $X, \phi: X \rightarrow(-\infty, \infty]$ be a proper, convex and lower semicontinuous function and $S: X \supseteq D(S) \rightarrow 2^{X^{*}}$ and $f^{*} \in X^{*}$. The variational inequality problem, denoted by VIP $\left(S, K, \phi, f^{*}\right)$, is said to be "solvable" in $D(S) \cap D(\partial \phi) \cap K$ if there exist $x_{0} \in \mathrm{D}(\mathrm{S}) \cap \mathrm{D}(\partial \phi) \cap \mathrm{K}$ and $v_{0}^{*} \in \mathrm{S} \mathrm{x}_{0}$ such that

$$
\left\langle v_{0}^{*}-f^{*}, x-x_{0}\right\rangle \geqslant \phi\left(x_{0}\right)-\phi(x), \quad \forall x \in K .
$$

The solvability of the problem $\operatorname{VIP}\left(S, K, \phi, f^{*}\right)$ is equivalent to the solvability of the inclusion problem

$$
S u+\partial \phi(u)+\partial I_{K}(u) \ni f^{*} \text { in } D(S) \cap D(\partial \phi) \cap K .
$$

The reader can compare inclusion and/or variational inequality results for $\left(S_{+}\right)$and/or pseudomonotone perturbations of maximal monotone operators in the papers by Kenmochi [20, 21], Le [24], Asfaw and Kartsatos [7, 8], Asfaw [2, 3, 5, 6] and the references therein.

Theorem 4.5 yields an existence result for a variational inequality problem. The maximal monotonicity condition on $A+\partial I_{K}+\partial \phi$ in Theorem 4.5 is achieved if $0 \in \mathrm{D}(A) \cap \stackrel{\circ}{\mathrm{K}} \cap \mathrm{D}(\stackrel{\circ}{\partial} \phi)$ (c.f., [31]).

Theorem 4.5. Let $\mathrm{U}$ be a nonempty and open subset of $\mathrm{X}$ and $\mathrm{K}$ be a nonempty, closed and convex subset of $\mathrm{X}$. Let $\mathrm{T}: \mathrm{X} \supseteq \mathrm{D}(\mathrm{T}) \rightarrow 2^{\mathrm{X}^{*}}$ and $\mathrm{A}: \mathrm{X} \supseteq \mathrm{D}(\mathrm{A}) \rightarrow 2^{\mathrm{X}^{*}}$ be maximal monotone operators such that $\mathrm{T}$ is quasibounded and of compact resolvents, and $\mathrm{C}: \mathrm{X} \supseteq \mathrm{D}(\mathrm{C}) \rightarrow \mathrm{X}^{*}$ be a bounded and continuous operator. Suppose $\phi: \mathrm{X} \rightarrow(-\infty, \infty]$ is a proper, convex and lower semicontinuous function such that $0 \in \mathrm{U} \cap \mathrm{K} \cap \mathrm{T}(0) \cap \mathrm{A}(0) \cap \partial \phi(0)$ and $\mathrm{A}+\partial \mathrm{I}_{\mathrm{K}}+\partial \phi$ is maximal monotone. Let $\mathrm{f}^{*} \in \mathrm{X}^{*}$. Then the problem $\operatorname{VIP}\left(\mathrm{T}+\mathrm{A}+\mathrm{C}, \mathrm{K}, \phi, \mathrm{f}^{*}\right)$ is solvable if exactly one of the following holds.

(i) $\mathrm{D}(\mathrm{A}) \subseteq \mathrm{D}(\mathrm{C})$ and there exists $\mathrm{R}>0$ such that

$$
\left\langle C x-f^{*}, x\right\rangle>0
$$

for all $x \in D(A) \cap \partial B_{R}(0)$.

(ii) $\mathrm{D}(\mathrm{C})=\overline{\mathrm{U}}$ and

$$
\left\langle C x-f^{*}, x\right\rangle>0
$$

for all $x \in \partial u$.

Proof. The proof can be completed using the operator $A+\partial I_{K}+\partial \phi$ instead of $A$ in the proof of Theorem 4.2. The details are omitted here.

We like to compare Theorem 4.5 with Theorem 2.7 due to Asfaw [6]. In [6], Asfaw proved the solvability of the problem $\operatorname{VIP}\left(T+C, K, \phi, f^{*}\right)$ provided that $\mathrm{D}(\mathrm{T}) \subseteq \mathrm{D}(\mathrm{C}), \mathrm{T}$ is quasibounded and of compact resolvent, $\partial \mathrm{I}_{\mathrm{K}}+\partial \phi$ is maximal monotone and there exist $\mathrm{u}_{0} \in \mathrm{D}(\partial \phi) \cap \mathrm{K}$ and $\mathrm{R}>0$ such that

$$
\left\langle u^{*}+C x-f^{*}, x-u_{0}\right\rangle>0,
$$


for all $x \in D(T) \cap \partial B_{R}(0)$ and $u^{*} \in T x$. One can see that [6, Theorem 2.7] follows from Theorem 4.5 (under condition (i)) if we use $\frac{1}{2} T$ instead of $T$ and $\frac{1}{2} T$ instead of $A$ with $u_{0}=0$. In addition the condition that $\partial \mathrm{I}_{K}+\partial \phi$ is maximal monotone is stronger than the maximal monotonicity of $\mathrm{T}+\partial \mathrm{I}_{\mathrm{K}}+\partial \phi$ because $\mathrm{T}$ is quasibouned with $0 \in \mathrm{D}(\mathrm{T})$, i.e., Theorem 4.5 used weaker conditions as compared to those used in [6] except using $\mathrm{u}_{0}=0$. In fact, condition (ii) in Theorem 4.5 provides a new result because no restriction is imposed on $\mathrm{D}(\mathrm{C})=\overline{\mathrm{U}}$ except that $\mathrm{U}$ is nonempty and open. In addition, Theorems 4.1, 4.2 and 4.5 hold if $\mathrm{D}(\mathrm{C})$ is nonempty with appropriate boundary conditions.

It is easy to see that for each $f^{*} \in X^{*}$ the problem $\operatorname{VIP}\left(T+A+C, K, \phi, f^{*}\right)$ is solvable if conditions of Theorem 4.5 are satisfied with $\mathrm{D}(\mathrm{A}) \subseteq \mathrm{D}(\mathrm{C})$ and (i) is replaced by the coercivity of $C$.

\section{An Application}

Suppose $p>1$ and $p^{\prime}$ is the conjugate exponent of $p$ (i.e., $\left.1 / p+1 / p^{\prime}=1\right), V=W_{0}^{1, p}(\Omega), X=$ $\mathrm{L}^{\mathrm{p}}(0, \mathrm{~T} ; \mathrm{V}), \mathrm{H}=\mathrm{L}^{2}(\Omega)$ and $\mathrm{X}^{*}=\mathrm{L}^{\mathrm{p}^{\prime}}\left(0, \mathrm{~T} ; \mathrm{V}^{*}\right)$, i.e., $\mathrm{V} \subseteq \mathrm{H} \subseteq \mathrm{V}^{*}$. For $\mathrm{u} \in \mathrm{X}$ and $v^{*} \in \mathrm{X}^{*}$, the norm of $u$ and $v^{*}$ is given by,

$$
\|\mathrm{u}\|_{\mathrm{X}}^{\mathrm{p}}=\int_{0}^{\mathrm{T}}\|\mathrm{u}(\mathrm{t})\|_{\mathrm{V}}^{\mathrm{p}} \mathrm{dt}, \quad \text { and } \quad\left\|v^{*}\right\|_{\mathrm{X}^{*}}^{\mathrm{p}^{\prime}}=\int_{0}^{\mathrm{T}}\left\|v^{*}(\mathrm{t})\right\|_{V^{*}}^{\mathrm{p}^{\prime}} \mathrm{dt} \text {. }
$$

In addition, the pairing between $w \in X^{*}$ (i.e., $w(t) \in V^{*}$ for all $t \in[0, T]$ ) and $u \in X$ (i.e., $u(t) \in V$ for all $\mathrm{t} \in[0, \mathrm{~T}])$, denoted by $\langle w, v\rangle$, is understood as

$$
\langle w, u\rangle=\int_{0}^{T}\langle w(t), u(t)\rangle_{v} d t
$$

In this section we shall apply the degree theories developed in Sections 2 and 3 to prove existence of solution for the variational inequality problem of finding $u \in D(L) \cap D(\partial \phi) \cap K$ such that

$$
\left\langle\mathrm{Lu}+\mathrm{Cu}-\mathrm{f}^{*}, v-\mathrm{u}\right\rangle \geqslant \Phi(\mathrm{u})-\Phi(v),
$$

for all $v \in K$, where $K$ is a nonempty, closed and convex subset of $X$ with $0 \in \stackrel{\circ}{K}$ and $L: X \supseteq D(L) \rightarrow X^{*}$ is defined by $L u=u^{\prime}$, where $u^{\prime}$ is understood in the sense of distributions, i.e.,

$$
\int_{0}^{T} u^{\prime}(t) \psi(t) d t=-\int_{0}^{T} u(t) \psi^{\prime}(t) d t, \psi \in C_{0}^{\infty}(0, T),
$$

with $\mathrm{D}(\mathrm{L})=\left\{\mathrm{u} \in \mathrm{X}: \mathrm{u}^{\prime} \in \mathrm{X}^{*}, \mathrm{u}(0)=\mathrm{u}(\mathrm{T})\right\}$ and

$$
\langle\mathrm{Lu}, \phi\rangle=\int_{0}^{T}\left\langle\mathrm{u}^{\prime}(\mathrm{t}), \phi(\mathrm{t})\right\rangle_{\mathrm{V}} \mathrm{dt}, \quad \mathrm{u} \in \mathrm{D}(\mathrm{L}), \quad \phi \in \mathrm{X}
$$

Let $C: X \rightarrow X^{*}$ be generated by the differential operator

$$
S u=-\sum_{i=1}^{N} \frac{\partial}{\partial x_{i}} a_{i}(x, u, \nabla u), \quad u \in X
$$

given by

$$
\langle C u, v\rangle=\sum_{i=1}^{N} \int_{Q} a_{i}(x, t, u(x, t), \nabla u(x, t)) \frac{\partial v}{\partial x_{i}} d x d t, \quad u \in X, \quad v \in X
$$

Suppose that

(a) for each $i=1,2, \cdots, N, a_{i}(x, t, \eta, \zeta)$ is Carathèodory function, i.e., $(x, t) \mapsto a_{i}(x, t, \eta, \zeta)$ is measurable for almost all $(\eta, \zeta) \in \mathbb{R}^{N+1}$ and $(\eta, \zeta) \mapsto a_{i}(x, t, \eta, \zeta)$ is continuous for almost all $(x, t) \in \Omega \times[0, T]$; 
(b) there exist $c_{1}>0$ and $k_{1} \in \mathrm{L}^{p^{\prime}}(\Omega \times[0, T])$ such that

$$
\left|a_{\mathfrak{i}}(x, t, \eta, \zeta)\right| \leqslant c_{1}\left(|\eta|^{p-1}+|\zeta|^{p-1}+k_{1}(x, t)\right),
$$

for all $(x, t) \in \Omega \times[0, T], \eta \in \mathbb{R}, \zeta \in \mathbb{R}^{N}$ and $i=1,2, \cdots, N$;

(c) there exists $\gamma>0$ such that

$$
\sum_{i=1}^{N} a_{i}(x, t, \eta, \zeta) \zeta_{i} \geqslant \gamma|\zeta|^{p},
$$

for all $(x, t) \in \Omega \times[0, T], \zeta \in \mathbb{R}^{N}$ and $i=1,2, \cdots, N$.

(d) $\Phi: X \rightarrow(-\infty, \infty]$ is a proper, convex and lower semicontinuous function of compact type (i.e., $\partial \Phi$ is of compact resolvents) and $\partial \Phi: X \supseteq \mathrm{D}(\partial \Phi) \rightarrow 2^{\mathrm{X}^{*}}$ is quasibounded (e.g., $0 \in \mathrm{D}(\dot{\partial} \Phi)$ ) such that $0 \in \partial \Phi(0)$.

Next we give the following existence theorem.

Theorem 5.1. Let $\mathrm{K}$ be a nonempty, closed and convex subset of $\mathrm{X}$ with $0 \in \stackrel{\circ}{\mathrm{K}}$ and, $\mathrm{L}$ and $\mathrm{C}$ be defined in (5.2) and (5.3), respectively. Suppose (a) through (d) hold. Then for each $\mathrm{f}^{*} \in \mathrm{X}^{*}$ problem (5.1) is solvable.

Proof. The operator $\mathrm{L}: \mathrm{X} \supseteq \mathrm{D}(\mathrm{L}) \rightarrow \mathrm{X}^{*}$ is known to be densely defined and maximal monotone. The proof of this fact and more details can be found in the book by Zeidler [34, pp. 354-918]. It is also true that condition (a) and (b) imply that the operator $\mathrm{C}: \mathrm{X}=\mathrm{D}(\mathrm{C}) \rightarrow \mathrm{X}^{*}$ is a bounded and continuous operator. The operator $L+\partial I_{K}$ is maximal monotone because $0 \in \stackrel{\circ}{K} \cap D(L) \neq \emptyset$ (c.f., [31]). We shall apply Corollary 4.3 of Theorem 4.2 using the operators $T=\partial \Phi, A=L+\partial I_{K}$ and $C$. It is sufficient to show that $C$ is coercive. Indeed condition (c) gives

$$
\langle\mathrm{Cu}, \mathrm{u}\rangle \geqslant \gamma \int_{0}^{T}\|\mathrm{u}(\mathrm{t})\|_{V}^{\mathrm{p}} \mathrm{dt}=\gamma\|u\|^{p},
$$

for all $u \in X$, i.e., we have

$$
\frac{\langle\mathrm{Cu}, \mathrm{u}\rangle}{\|\mathrm{u}\|} \geqslant\|\mathrm{u}\|^{\mathrm{p}-1}
$$

for all $x \in X \backslash\{0\}$. Since $p>1$, letting $\|u\| \rightarrow \infty$ implies the coercivity of $C$. Then Corollary 4.3 yields the surjectivity of the operator $T+A+C=\partial \Phi+L+\partial I_{K}+C$, i.e., for each $f^{*} \in X^{*}$ we get the solvability of $\operatorname{VIP}\left(\mathrm{L}+\mathrm{C}, \mathrm{K}, \Phi, \mathrm{f}^{*}\right)$ in $\mathrm{D}(\mathrm{L}) \cap \mathrm{D}(\partial \Phi) \cap \mathrm{K}$, i.e., (5.1) is solvable. The proof is completed.

It can been seen that the proof of Theorem 4.5 does not require the operator $C$ to satisfy pseudomonotonicity condition. It is essential to highlight here that pseudomonotonicity of the operator $C$ is achieved if (a) and (b) are satisfied, and

$$
\sum_{i=1}^{N}\left(a_{i}(x, t, \eta, \zeta)-a_{i}\left(x, t, \eta, \zeta^{\prime}\right)\left(\zeta_{i}-\zeta_{i}^{\prime}\right)>0,\right.
$$

for all $(x, t, \eta) \in \Omega \times[0, T] \times \mathbb{R}, \zeta \in \mathbb{R}^{N}$ and $\zeta^{\prime} \in \mathbb{R}^{N}$. In addition, $C$ satisfies the $\left(S_{+}\right)$condition if (a), (b), (c) and (5.4) hold. Notice here that condition (5.4) is not required in the proof of Theorem 4.5 because of the resolvent compactness condition on $\partial \Phi$. The reader can find further details about differential operators of type $\left(S_{+}\right)$in the paper by Berkovits and Mustonen [10], Hu and Papageorgiou [18], Landes and Mustonen [23], and the references therein. 


\section{Acknowledgment}

I would like to thank the editor and anonymous referee(s) for careful reading of the manuscript. I am thankful to Virginia Tech for paying the article processing charge.

\section{References}

[1] D. R. Adhikari, A. G. Kartsatos, Strongly quasibounded maximal monotone perturbations for the Berkovits-Mustonen topological degree theory, J. Math. Anal. Appl., 348 (2008), 122-136. 1

[2] T. M. Asfaw, New variational inequality and surjectivity theories for perturbed noncoercive operators and application to nonlinear problems, Adv. Math. Sci. Appl., 24 (2014), 611-668. 4

[3] T. M. Asfaw, A new topological degree theory for pseudomonotone perturbations of the sum of two maximal monotone operators and applications, J. Math. Anal. Appl., 434 (2016), 967-1006. 1, 1, 4

[4] T. M. Asfaw, A new topological degree theory for perturbations of demicontinuous operators and applications to nonlinear equations with nonmonotone nonlinearities, J. Funct. Spaces, 2016 (2016), 15 pages. 1, 1

[5] T. M. Asfaw, A degree theory for compact perturbations of monotone type operators in reflexive Banach spaces, Abstr. Appl. Anal., 2017 (2017), 13 pages. 1, 1, 4

[6] T. M. Asfaw, A variational inequality theory for constrained problems in reflexive Banach spaces, Adv. Oper. Theory, 4 (2019), 462-480. 1, 4, 4

[7] T. M. Asfaw, A. G. Kartsatos, A Browder topological degree theory for multivalued pseudomonotone perturbations of maximal monotone operators in reflexive Banach spaces, Adv. Math. Sci. Appl., 22 (2012), 91-148. 1, 1, 4

[8] T. M. Asfaw, A. G. Kartsatos, Variational inequalities for perturbations of maximal monotone operators in reflexive Banach spaces, Tohoku Math. J. (2), 66 (2014), 171-203. 4

[9] V. Barbu, Nonlinear Differential Equations of Monotone Types in Banach Spaces, Springer, New York, (2010). 1.1, 1

[10] J. Berkovits, V. Mustonen, On the topological degree for mappings of monotone type, Nonlinear Anal., 10 (1986), 13731383. $1,1,5$

[11] H. Brézis, M. G. Crandall, A. Pazy, Perturbations of nonlinear maximal monotone sets in Banach spaces, Comm. Pure Appl. Math., 23 (1970), 123-144. 2

[12] L. E. J. Brouwer, Über Abbildung von Mannigfaltigkeiten, Math. Ann., 71 (1912), 97-115. 1

[13] F. E. Browder, Nonlinear operators and nonlinear equations of evolution in Banach spaces, Proc. Sympos. Pure Math., 18 (1976), 1-308. 1

[14] F. E. Browder, Degree of mapping for nonlinear mappings of monotone type, Proc. Nat. Acad. Sci., 80 (1983), 1771-1773. 1

[15] F. E. Browder, Degree of mapping for nonlinear mappings of monotone type; Strongly nonlinear mapping, Proc. Nat. Acad. Sci. U.S.A., 80 (1983), 2408-2409. 1

[16] F. E. Browder, Degree of mapping for nonlinear mappings of monotone type: densely defined mapping, Proc. Nat. Acad. Sci. U.S.A., 80 (1983), 2405-2407. 1, 1

[17] F. E. Browder, P. Hess, Nonlinear mappings of monotone type in Banach spaces, J. Funct. Anal., 11 (1972), 251-294. 1

[18] S. C. Hu, N. S. Papageorgiou, Generalization of Browder's degree theory, Trans. Amer. Math. Soc., 347 (1995), $233-259$. $1,1,5$

[19] A. G. Kartsatos, I. V. Skrypnik, Topological degree theories for densely defined mappings involving operators of type $\left(\mathrm{S}_{+}\right)$, Adv. Differential Equations, 4 (1999), 413-456. 1, 1

[20] N. Kenmochi, Nonlinear operators of monotone type in reflexive Banach spaces and nonlinear perturbations, Hiroshima Math. J., 4 (1974), 229-263. 4

[21] N. Kenmochi, Monotonicity and compactness methods for nonlinear variational inequalities, in: Handbook of differential equations: stationary partial differential equations, 2007 (2007), 203-298. 4

[22] J. Kobayashi, M. Otani, Topological degree for $(\mathrm{S})_{+}$-mappings with maximal monotone perturbations and its applications to variational inequalities, Nonlinear Anal., 59 (2004), 147-172. 1, 1

[23] R. Landes, V. Mustonen, On pseudomonotone operators and nonlinear noncoercive variational problems on unbounded domain, Math. Ann., 248 (1980), 241-246. 5

[24] V. K. Le, A range and existence theorem for pseudomonotone perturbations of maximal monotone operators, Proc. Amer. Math. Soc., 139 (2011), 1645-1658. 4

[25] J. Leray, J. Schauder, Topologie et équations fonctionnelles, Ann. Sci. École Norm. Sup. (3), 51 (1934), 45-78. 1

[26] N. G. Lloyd, Degree Theory, Cambridge University Press, New York, (1978).

[27] J. Mawhin, Leray-Schauder degree: a half a century of extensions and applications, Topol. Methods Nonlinear Anal., 14 (1999), 195-228. 1 1

[28] M. Nagumo, Degree of mapping in convex linear topological spaces, Amer. J. Math., 73 (1951), 497-511. 1

[29] D. O'Regan, Y. J. Cho, Y.-Q. Chen, Topological Degree Theory and Applications, Chapman and Hall/CRC, Boca Raton, (2006). 1 
[30] D. Pascali, S. Sburlan, Nonlinear Mappings of Monotone Type, Sijthoff \& Noordhoff International Publishers, Bucharest, (1978). 1

[31] R. T. Rockafellar, On the maximality of sums of nonlinear monotone operators, Trans. Amer. Math. Soc., 149 (1970), 75-88. 4, 5

[32] R. E. Showalter, Monotone operators in Banach space and nonlinear partial differential equations, Amer. Math. Soc., Providence, (1997). 1

[33] I. I. Vrabie, Compactness methods for nonlinear evolutions, Longman Scientific \& Technical, Harlow, (1995). 1

[34] E. Zeidler, Nonlinear Functional Analysis and Its Applications, Springer-Verlag, New York, (1990). 1, 1, 5 